\title{
Dietary substitution of SFA with MUFA within high-fat diets attenuates hyperinsulinaemia and pancreatic islet dysfunction
}

Jessica C. Ralston ${ }^{1}$, Marie-Sophie Nguyen-Tu² ${ }^{2}$ Claire L. Lyons ${ }^{1}$, Aoife A. Cooke ${ }^{1}$, Aoife M. Murphy ${ }^{1}$, Aidan Falvey ${ }^{1}$, Orla M. Finucane ${ }^{1}$, Fiona C. McGillicuddy ${ }^{1,3}$, Guy A. Rutter ${ }^{2}$ and Helen M. Roche ${ }^{1,3,4 *}$

${ }^{1}$ Nutrigenomics Research Group, UCD Conway Institute of Biomolecular Research, and School of Public Health, Physiotherapy and Sports Science, University College Dublin, Belfield, Dublin 4, Republic of Ireland

${ }^{2}$ Section of Cell Biology and Functional Genomics, Department of Medicine, Imperial College London, Hammersmith Hospital, London W12 ONN, UK

${ }^{3}$ Diabetes Complications Research Centre, UCD Conway Institute of Biomolecular and Biomedical Research, University College Dublin, Dublin, Republic of Ireland

${ }^{4}$ Institute for Global Food Security, School of Biological Sciences, Queen's University Belfast, Belfast BT7 1NN, UK

(Submitted 31 May 2019 - Final revision received 13 February 2020 - Accepted 21 February 2020 - First published online 3 March 2020)

\section{Abstract}

Preliminary evidence has suggested that high-fat diets (HFD) enriched with SFA, but not MUFA, promote hyperinsulinaemia and pancreatic hypertrophy with insulin resistance. The objective of this study was to determine whether the substitution of dietary MUFA within a HFD could attenuate the progression of pancreatic islet dysfunction seen with prolonged SFA-HFD. For 32 weeks, C57BL/6J mice were fed either: (1) low-fat diet, (2) SFA-HFD or (3) SFA-HFD for 16 weeks, then switched to MUFA-HFD for 16 weeks (SFA-to-MUFA-HFD). Fasting insulin was assessed throughout the study; islets were isolated following the intervention. Substituting SFA with MUFA-HFD prevented the progression of hyperinsulinaemia observed in SFA-HFD mice $(P<0.001)$. Glucose-stimulated insulin secretion from isolated islets was reduced by SFA-HFD, yet not fully affected by SFA-to-MUFA-HFD. Markers of $\beta$-cell identity (Ins2, $N k x 6.1, N g n 3, R f x \sigma, P d x 1$ and $P a x G$ ) were reduced, and islet inflammation was increased (IL-1 $\beta, 3 \cdot 0$-fold, $P=0.007$; CD68, 2-9-fold, $P=0 \cdot 001 ; I l-6,1 \cdot 1$-fold, $P=0.437$ ) in SFA-HFD - effects not seen with SFA-to-MUFA-HFD. Switching to MUFA-HFD can partly attenuate the progression of SFA-HFD-induced hyperinsulinaemia, pancreatic inflammation and impairments in $\beta$-cell function. While further work is required from a mechanistic perspective, dietary fat may mediate its effect in an IL-1 $\beta$-AMP-activated protein kinase $\alpha 1$-dependent fashion. Future work should assess the potential translation of the modulation of metabolic inflammation in man.

Key words: Diet-induced obesity: Insulin secretion: Islets: $\beta$-Cell identity: Metabolic inflammation

Hyperinsulinaemia is a highly pathogenic physiological state, characteristic of obesity and insulin resistance, preceding the onset of pancreatic islet dysfunction and overt type 2 diabetes $^{(1)}$. High-fat diets (HFD) promote obesity, hyperinsulinaemia and metabolic inflammation ${ }^{(2)}$. However the composition of fatty acids within HFD alters the impact of dietary fat on insulin biology ${ }^{(3,4)}$. Feeding SFA-enriched HFD (SFA-HFD) primed and activated nucleotide-binding domain, leucine-rich-containing family, pyrin domain-containing-3 (NLRP3)-mediated IL-1 $\beta$ activation and insulin resistance in adipose tissue, concomitant with hyperinsulinaemia and pancreatic hypertrophy compared with MUFA-enriched $\mathrm{HFD}^{(5)}$. Although previous work from our team suggested that switching from SFA to MUFA-HFD (SFA-to-MUFA-HFD) attenuated the increment in fasting insulin levels ${ }^{(5)}$, that study was not ideal in that a healthy, low-fat-diet (LFD) control group phenotype was not within the study design. Other studies have reported that replacing dietary SFA (palmitate) with high MUFA (oleate) intake reduced inflammatory cytokine secretion $^{(6)}$ and improved insulin sensitivity in women ${ }^{(7)}$. Importantly, understanding of putative, inflammatory-related mechanisms underpinning the differential effects of SFA-HFD $v$. MUFA-HFD on pancreatic islet function has remained elusive ${ }^{(5)}$.

Inflammation in metabolic tissues plays a critical role in peripheral insulin resistance and is influenced by dietary constituents, including fatty acids ${ }^{(2,3)}$. In obesity, proinflammatory cytokines, including IL- $1 \beta$ and IL- 6 , disrupt normal cellular signalling and metabolic pathways ${ }^{(2,8)}$. Pancreatic inflammation

Abbreviations: AMPK, AMP-activated protein kinase $\alpha 1$; HFD, high-fat diet; LFD, low-fat diet; NLRP3, nucleotide-binding domain, leucine-rich-containing family, pyrin domain-containing-3.

* Corresponding author: Helen M. Roche, fax +353-1-716-6701, email helen.roche@ucd.ie 
reduces islet insulin secretion, and chronically elevated IL-1 $\beta$ production/secretion promotes $\beta$-cell apoptosis and dysfunction $^{(9-11)}$. Masters et al. ${ }^{(12)}$ also demonstrated that amylin (the main constituent of pancreatic amyloid deposits in type 2 diabetes) activated NLRP3-mediated IL-1 $\beta$ production. Moreover, palmitate is a potent SFA, which is well known to prime and activate IL-1 $\beta$ in an NLRP3-dependent fashion ${ }^{(13,14)}$. Interesting in vitro $\beta$-cell studies have shown that MUFA exposure can prevent SFA-induced apoptosis and impairments in cell proliferation ${ }^{(15)}$. These varying effects of SFA $v$. MUFA warrant further investigation.

The present study has addressed the hypothesis that substitution of dietary MUFA attenuates the adverse effects of SFA-HFD on pancreatic islet function and differentiation. We used a regression feeding model where mice switched from SFA-HFD to MUFA-HFD (i.e. SFA-to-MUFA-HFD) were compared with mice maintained on an SFA-HFD and to healthy LFD control mice, an important extension from previous work in the field. We show that SFA-HFD reduced markers of pancreatic $\beta$-cell identity (e.g. Ins $2, N k x 6.1, N g n 3, R f x 6, P d x 1$ and Pax $)^{(16)}$, coincident with increased inflammation and in vivo hyperinsulinaemia; these effects were either partially or fully attenuated in SFA-to-MUFA-HFD mice. This study provides important evidence that dietary MUFA can offset the detrimental effects of prolonged SFA-HFD on pancreatic function and inflammation. Our findings also further highlight the importance of examining the type of dietary fat composition, rather than quantity alone, when considering overall metabolic health.

\section{Materials and methods}

\section{Materials and cell culture reagents}

Cell culture solutions were purchased from Lonza. All other reagents, unless otherwise stated, were purchased from Sigma-Aldrich.

\section{Animals}

Male C57BL/6J mice (aged 7-9weeks) were purchased from Harlan UK Ltd. Ethical approval was obtained from the University College Dublin Ethics Committee (P15-35), and mice were maintained according to the regulations of the Health Products Regulatory Authority (Directive 2010/63/EU and Irish Statutory Instrument 543 of 2012). Mice were randomly assigned to treatment groups and fed one of three study diets: (1) LFD (10\% energy; $n$ 10) for 32 weeks; (2) SFA-based HFD for 32 weeks (SFA-HFD; $45 \%$ energy; $n$ 10); or (3) SFA-based diet for 16 weeks followed by a MUFA-based diet for an additional 16 weeks (SFA-to-MUFA-HFD; $45 \%$ energy; $n$ 10). The experimental model is depicted in online Supplementary Fig. S1. All study diets were purchased from Research Diets Inc. (catalogue nos. D12450B, D07081501, D07062503, respectively) and represent a reasonable amount of dietary fat expected in the human population. Diet composition and fatty acid profiles are presented in online Supplementary Tables S1 and S2. Body weight and food intake were monitored weekly. Upon completion of the study, mice were euthanised by cervical dislocation.

\section{Metabolic phenotyping of mice}

Insulin secretory response was assessed in overnight-fasted mice, where tail blood samples were collected at indicated time points after injection of glucose $(25 \% \mathrm{w} / \mathrm{v}, 1.5 \mathrm{~g} / \mathrm{kg}$ intraperitoneally (i.p.); B. Braun Medical). Insulin concentration was measured by ELISA (Crystal Chem). For glucose and insulin tolerance tests, mice were fasted for $6 \mathrm{~h}$ prior to the injection of glucose $(25 \% \mathrm{w} / \mathrm{v}, 1.5 \mathrm{~g} / \mathrm{kg}$ i.p. $)$ or insulin $(0.5 \mathrm{U} / \mathrm{kg}$; Actrapid, Novo Nordisk), respectively. Glucose levels were monitored at indicated time points using a blood glucometer from Accu-Check (Roche). Throughout the study, fasting insulin and glucose levels were also measured by ELISA or glucometer, respectively, at weeks 0, 16, 20, 24 and 32 .

\section{Pancreatic immunostaining and isolation of islets}

Following euthanasia, either pancreatic immunostaining or islet isolation was conducted. For immunostaining, mouse pancreata were removed and fixed in $10 \%$ neutral balanced formalin prior to dehydration and paraffin-embedding. Tissues were sectioned to obtain 8- $\mu \mathrm{m}$ slices using a microtome (Leica RM 2135). Sections were rehydrated, followed by antigen retrieval and the addition of a hydrophobic barrier using a dako pen. Samples were blocked with $10 \%$ bovine serum albumin, washed and incubated in the dark at $4^{\circ} \mathrm{C}$ with primary antibodies IL- $1 \beta$ (Abcam Ab9722; $1 \mu \mathrm{g} / \mathrm{ml}$ ) and CD68 (Abcam Ab53444; $2 \mu \mathrm{g} / \mathrm{ml}$ ). The next day, fluorescent secondary antibodies were added for $1 \mathrm{~h}$, with the nuclear stain 4',6-diamidino-2-phenylindole (DAPI) included during the final $20 \mathrm{~min}$ of incubation. Sections were imaged using a Zeiss Axio Imager M1 and quantified using AxioVision (Zeiss) and ImageJ software.

For islet isolation, the pancreas was perfused through the pancreatic duct with collagenase (SERVA) and the tissue digested as previously described ${ }^{(17)}$. Islet separation was completed using two Histopaque density gradients (Sigma-Aldrich), and islets were hand-picked and cultured in Roswell Park Memorial Institute (RPMI) medium supplemented with $10 \%$ heat-inactivated fetal bovine serum and $1 \%$ penicillin-streptomycin ${ }^{(17,18)}$.

\section{Assessment of islet function}

Glucose-stimulated insulin secretion from isolated islets was assessed as described previously ${ }^{(18-20)}$. Briefly, after culturing islets in Krebs-Ringer-HEPES-bicarbonate solution (10 mm HEPES (4-(2-hydroxyethyl)-1-piperazineethanesulfonic acid), $140 \mathrm{~mm} \mathrm{NaCl}, 3.6 \mathrm{~mm} \mathrm{KCl}, 2 \mathrm{~mm} \mathrm{NaHCO}_{3}, 0.5 \mathrm{~mm} \mathrm{NaH} \mathrm{PO}_{4}$, $0.5 \mathrm{~mm} \mathrm{MgSO}_{4}, 1.5 \mathrm{~mm} \mathrm{CaCl}_{2}$, and $0.1 \%$ bovine serum albumin, pre-equilibrated with $95 \%: 5 \% \mathrm{O}_{2}: \mathrm{CO}_{2}$ and $\mathrm{pH} 7.4$ ) with $6 \mathrm{~mm}$ glucose for $1 \mathrm{~h}$ at $37^{\circ} \mathrm{C}$, batches of ten islets per mouse were then transferred to Krebs-Ringer-bicarbonate-HEPES supplemented with either low $(6 \mathrm{~mm})$ or high $(17 \mathrm{~mm})$ glucose for $30 \mathrm{~min}$ at $37^{\circ}$ $C^{(19,20)}$. Secreted and total insulin fractions were analysed by ELISA. The insulin secretion stimulatory index was obtained as a ratio of insulin secreted under high/low glucose conditions.

\section{Real-time RT-PCR}

Whole liver tissue was collected after sacrifice, snap-frozen in liquid nitrogen and stored at $-80^{\circ} \mathrm{C}$ prior to tissue 
homogenisation. Total RNA from islets or liver was extracted using TriReagent (Sigma-Aldrich) as per manufacturer's instructions, and quantified using a Nanodrop (ThermoFisher Scientific) $)^{(5)}$. Single-stranded complementary DNA was synthesised from $1 \mu \mathrm{g}$ of total RNA using a high-capacity complementary DNA Reverse Transcription Kit (ThermoFisher Scientific), and real-time RT-PCR was conducted using a QuantStudio 7 Flex RT-PCR system and Taqman Gene Expression Master Mix with SYBR Green (ThermoFisher ABI). Primer details are provided in online Supplementary Table S3. Changes in gene expression were determined using the $\Delta \Delta \mathrm{Ct}$ normalisation and quantification $\operatorname{method}^{(21)}$, where $\beta$-actin $(A c t b)$ and 18 s were used as housekeeping genes for islets and liver, respectively. Both reference genes were highly stable in their respective tissues $(<2.4$ and $<2.7 \%$ variability). All primers were purchased from Applied Biosystems (ThermoFisher Scientific).

\section{Statistical analyses}

Data are reported as mean values with their standard errors. Sample size for detecting a significant difference between groups (insulin concentration as main parameter) was calculated using G*Power (3.1.9.2) assuming an effect size of $1 \cdot 27$, a type I error of $0 \cdot 05$ (two tails) and statistical power of $0 \cdot 80$. To analyse metabolic phenotype data with multiple time points (insulin secretion response, glucose tolerance test, insulin tolerance test, changes in fasted insulin and homeostasis model assessment of insulin resistance over time $\left.{ }^{(22)}\right)$, we performed two-way repeatedmeasures ANOVA to test for differences between groups. When an ANOVA was significant, Bonferroni-corrected post hoc comparisons were examined. AUC analysis was performed on curves from the insulin secretion response using GraphPad Prism 5 software. For between-group comparisons at a single time point, one-way ANOVA was performed with Bonferronicorrected post hoc comparisons when an ANOVA was significant. GraphPad Prism 5 was used for all statistical analyses. A $P$ value $<0.05$ was considered statistically significant, with significant comparisons described in the captions of all figures.

\section{Results}

Switching to MUFA-high-fat diet attenuated hyperinsulinaemia regardless of changes in body weight

In order to determine whether MUFA-HFD may offset the impact of SFA-HFD, we used a regression feeding model wherein mice were first fed an SFA-HFD for 16 weeks to induce obesity and hyperinsulinaemia. Half of the SFA-HFD mice were then switched to MUFA-HFD, and this group (SFA-to-MUFA-HFD) was compared with SFA-HFD and age-matched LFD control mice. Interestingly, switching from SFA-to-MUFA-HFD after 16-week HFD prevented further elevations in fasting insulin levels over time as was observed in mice maintained on SFA-HFD $(P<0 \cdot 001 ; n$ 15-30; Fig. 1(a)). Nonetheless, the SFA-to-MUFAHFD group remained hyperinsulinaemic compared with the LFD group, indicating that MUFA intervention could block further progression of disease but did not completely regress the adverse phenotype. Differences in circulating insulin between HFD groups remained significant after weight-matching mice (data not shown). Furthermore, switching from SFA-to-MUFA-HFD also improved homeostasis model assessment of insulin resistance and homeostasis model assessment of insulin sensitivity over time (Fig. 1(a) and (c)). There were no significant differences in homeostasis model assessment- $\beta$-cell function between SFA-HFD and SFA-toMUFA-HFD groups (data not shown).

Interestingly, upon completion of the study (week 32), the insulin secretion response to glucose injection was attenuated in the SFA-to-MUFA-HFD group compared with SFA-HFD $(P<0 \cdot 001 ; n 15-30 ;$ Fig. $1(\mathrm{~d}))$. Both HFD groups had an elevated insulin secretion response compared with LFD. Similarly, the insulin secretion response AUC was significantly reduced in SFA-to-MUFA-HFD mice, compared with feeding the SFA-HFD alone $(P<0 \cdot 0001$; Fig. 1(e)). There was no difference in the insulin secretion response or corresponding AUC at week 16, prior to the dietary switch (data not shown). In terms of adjusting for differences in fasting insulin concentrations, the incremental insulin secretion AUC in SFA-to-MUFA-HFD mice was similar to the LFD, with both groups being significantly lower than SFA-HFD mice $(P=0.0013$ and 0.0007 , respectively; $n$ 10-11; Fig. 1(f)). Also, in terms of weight gain, at week 16 prior to the dietary switch, there were no significant differences in body weight between mice assigned to SFA-to-MUFA-HFD or maintained on SFA-HFD, nor were there differences in caloric intake (online Supplementary Fig. 2(e) and (f)). However, by the end of the intervention (week 32), SFA-HFD mice had gained more weight than SFA-to-MUFA-HFD mice $(P=0.0008 ; n 10$; Fig. 1(g)). Nevertheless, despite weight matching, the insulin secretion response remained more profound in SFA-HFD mice compared with the SFA-to-MUFA-HFD group (Fig. 1(h)). Glucose and insulin tolerance tests were not different between SFA-HFD and SFA-to-MUFA-HFD groups (online Supplementary Fig. S2(a) and (b)), indicating no difference in insulin resistance between HFD groups.

Switching from SFA to MUFA-high-fat diet attenuated the adverse effects of SFA-high-fat diet on markers of $\beta$-cell function, metabolism and differentiation

To examine whether the dietary fat composition induced changes in islet function, we assessed glucose-stimulated insulin secretion from isolated islets. Interestingly, the insulin stimulatory index was markedly reduced in islets isolated from SFA-HFD mice, compared with LFD mice $(P=0 \cdot 002 ; n$ 10; Fig. $2(\mathrm{a})$ ). However, despite the onset of obesity in both HFD groups, the reduction in insulin stimulatory index was not as profound in islets from SFA-to-MUFA-HFD mice $(P=0.078$; $n$ 10; Fig. 2(a)). There were no significant differences in basal islet insulin secretion between diet groups (online Supplementary Fig. S2(c)).

In terms of understanding the molecular perturbations induced in pancreatic islets, Ins 2 mRNA expression was significantly reduced after SFA-HFD ( $P=0.003 v$. LFD; $n$ 10; Fig. 2(b)), but this was preserved by switching mice from SFA-to-MUFAHFD. Similarly, islet Ampk (Prkaa1) mRNA expression was markedly reduced in SFA-HFD mice, compared with the LFD group $(P=0.004)$, an effect that was partially prevented by 

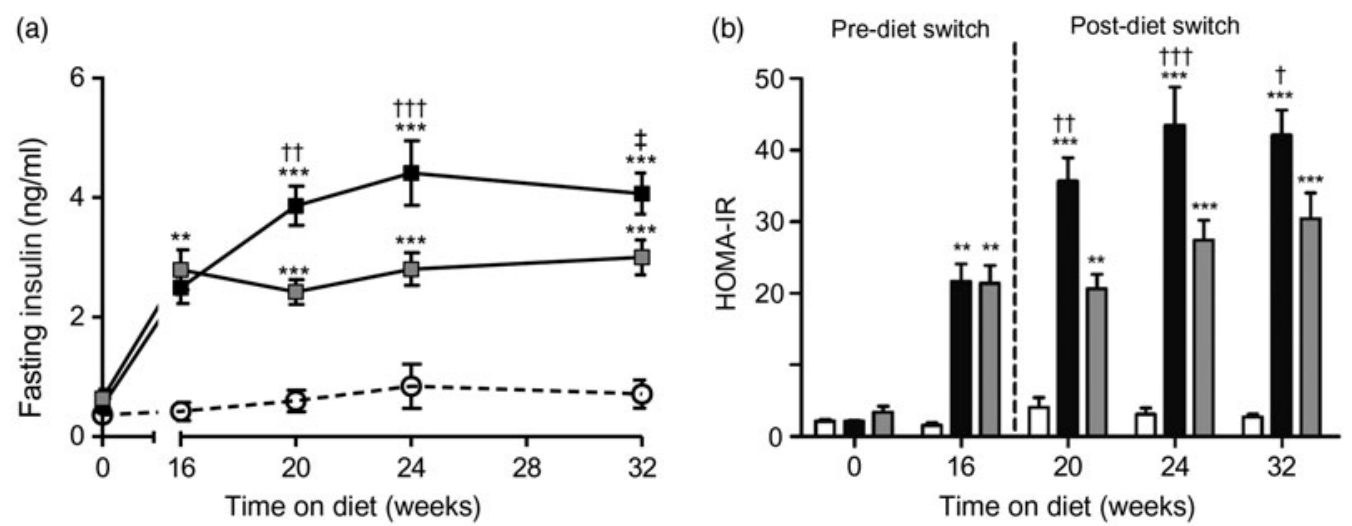

(c)

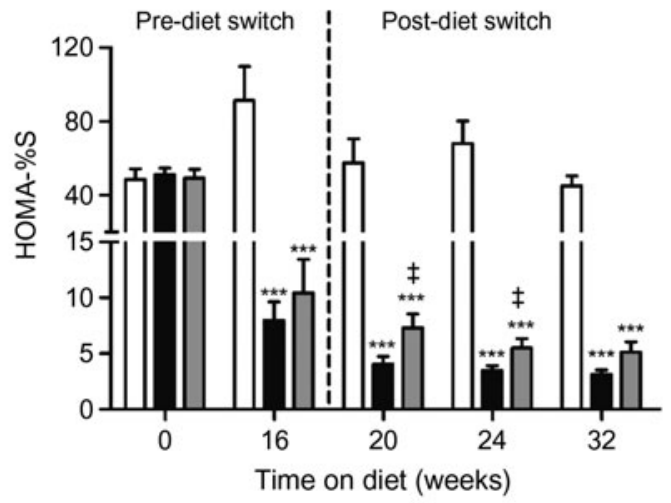

(d)

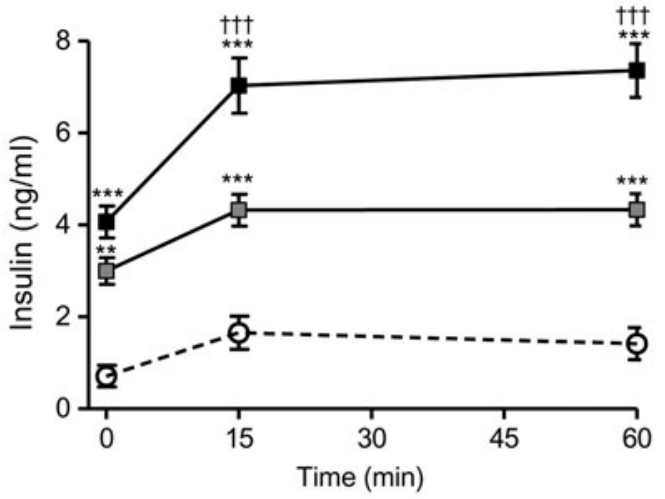

(e)

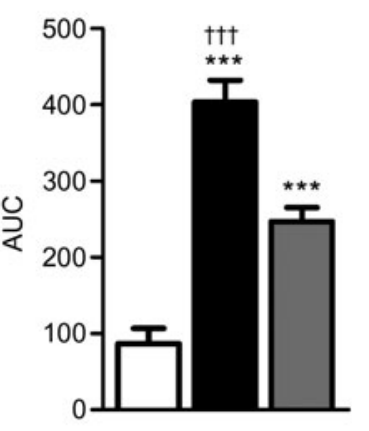

(f)

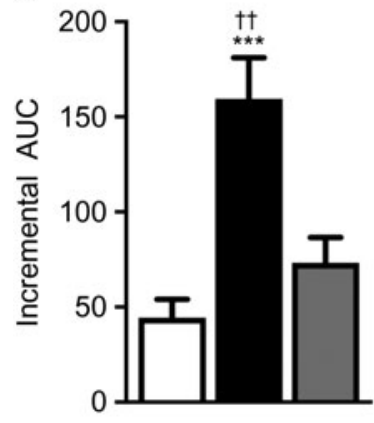

(g)

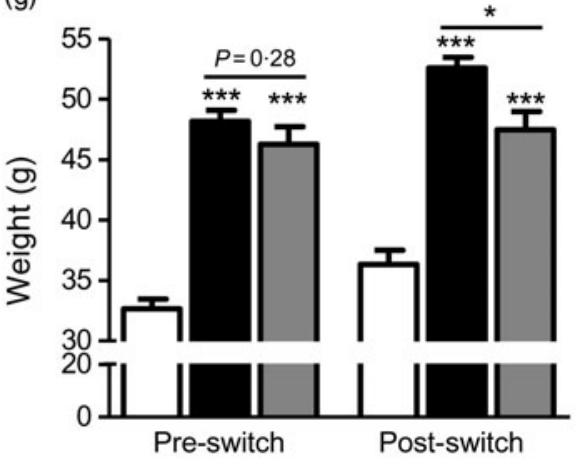

(h)

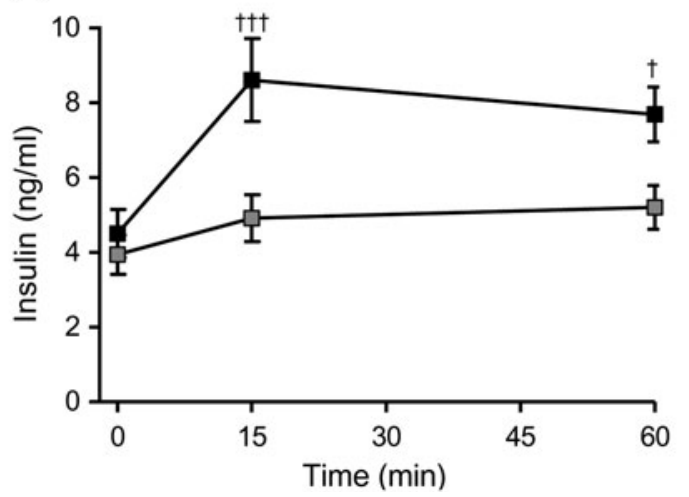

Fig. 1. Effects of a dietary switch from SFA-to-MUFA-high-fat diet (HFD) on metabolic phenotype. Fasting insulin levels (a), homeostatic model assessment of insulin resistance (HOMA-IR) (b) and homeostatic model assessment of insulin sensitivity (HOMA-\%S) (c) were tracked throughout the dietary intervention. Insulin secretion response was examined in overnight-fasted mice ( $(15-30)$ with intraperitoneal injection of $1.5 \mathrm{~g} / \mathrm{kg}$ glucose (d), and corresponding AUC (e) and incremental AUC (f) are shown. Mice weights are indicated in (g). Given a difference in body weight at week 32, the insulin secretion response was also assessed in weight-matched groups (h). (a-h), ${ }^{*} P<0.05,{ }^{* *} P<0.01,{ }^{* * *} P<0.001 v$. low-fat diet (LFD); $\dagger P<0.05$, $\dagger \dagger P<0.01, \dagger^{\circ} \dagger P<0.001$, SFA-HFD $v$. SFA-to-MUFA-HFD; by two-way (a-d, h) or one-way (e-g) ANOVA with Bonferroni post hoc comparisons; $\ddagger P<0.05$ SFA-HFD $v$. SFA-to-MUFA-HFD by unpaired Student's $t$ test. (a, d, h) $-\Theta-$-, LFD; $-\rightarrow-$, SFA-HFD; $-\square-$, SFA-to-MUFA-HFD; (b, c, e, f, g) $\square$, LFD; $\square$, SFA-HFD; $\square$, SFA-to-MUFA-HFD.

switching from SFA-to-MUFA-HFD ( $P=0.029 ; n$ 10; Fig. 2(b)). However, despite these differences, the expression of $L d h a$ (a $\beta$-cell 'disallowed' or selectively repressed gene sensitive to $A m p k^{(23-25)}$ ) was not significantly changed (Fig. 2(b)).

We next assessed whether switching from SFA-to-MUFAHFD could alter the expression of $\beta$-cell-enriched transcription factors and key genes that maintain $\beta$-cell identity. The expressions of $N k x 6.1, N g n 3$ and $R f x 6$ were all significantly reduced in mice in the SFA-HFD group compared with LFD $(P=0.043$,
0.042 and 0.046 , respectively; $n$ 10; Fig. 2(c)). Conversely, the expression of this panel of genes was significantly elevated in SFA-to-MUFA-HFD mice compared with SFA-HFD mice, to levels that were not significantly different from LFD mice $(P=0.017,0.007$ and $0.011 v$. SFA-HFD for Nkx6.1, Ngn3 and $R f x \sigma$, respectively; $n$ 10; Fig. 2(c)). $P d x 1$ and Pax 6 mRNA levels showed a similar response, albeit not statistically significant. Dietary modifications did not alter islet MafA expression. 
(a)

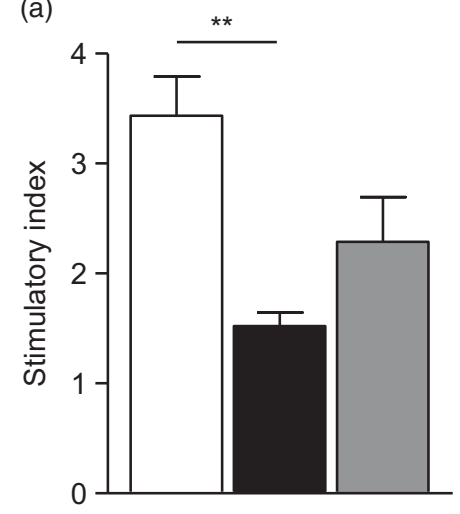

(b)

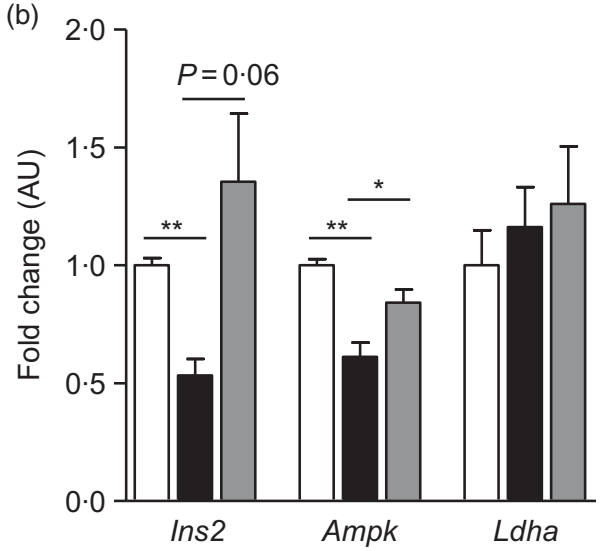

(c)

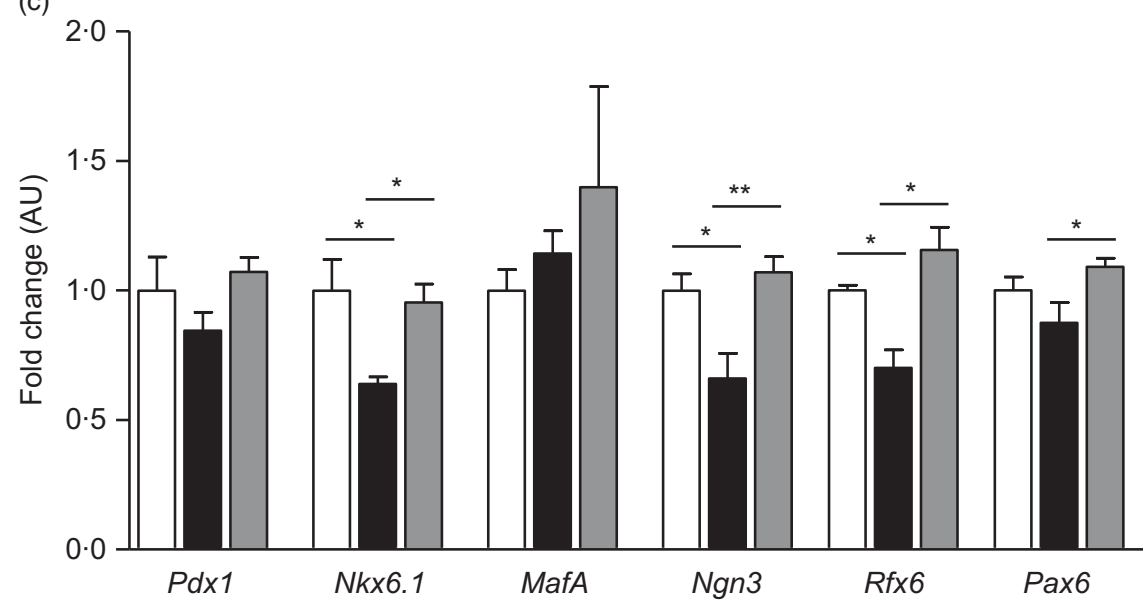

Fig. 2. Effects of a dietary switch from SFA-to-MUFA-high-fat diet (HFD) on pancreatic islet function. Insulin stimulatory index (a), and markers of $\beta$-cell identity and differentiation (b and $\mathrm{c}$ ) were examined. ${ }^{*} P<0.05,{ }^{\star \star} P<0.01,{ }^{* * *} P<0.001$ between indicated comparisons by one-way ANOVA with Bonferroni post hoc comparisons. AU, arbitrary units; Ins2, insulin II; Ampk, AMP-activated protein kinase $\alpha 1$; Ldha, lactate dehydrogenase A; Pdx1, pancreatic and duodenal homeobox 1; Nkx6.1, NK6 homeobox 1; MafA, pancreatic $\beta$-cell-specific transcriptional activator; Ngn3, neurogenin 3; Rfx6, regulatory factor X6; Pax6, paired box gene 6. (a-c) $\square$, low-fat diet; , SFA-HFD; $\square$, SFA-to-MUFA-HFD.

The liver plays a key role in insulin clearance and degradation. Consequently, we assessed hepatic Ceacam1 expression, as the deletion of this gene causes hyperinsulinaemia due to impaired insulin clearance in addition to increased lipogenic gene expression and insulin resistance ${ }^{(26,27)}$. Hepatic Ceacam 1 was most reduced by SFA-HFD, an effect that was not fully affected by switching to MUFA-HFD (Fig. 3). Similarly, hepatic Irs-2 mRNA was lowered by both HFD irrespective of fatty acid composition (Fig. 3). Other lipogenic genes, including Acc- $\alpha$, Fasn and $S c d 1$, were not markedly altered between diets (online Supplementary Fig. S2(d)).

\section{Pancreatic inflammatory markers were significantly} reduced in mice switched from SFA-to-MUFA-high-fat diet

Palmitate is a potent trigger of IL- $1 \beta$ signalling, which can promote pancreatic inflammation and $\beta$-cell dysfunction ${ }^{(28)}$. Immunostaining results demonstrated enhanced expression of both IL-1 $\beta(P=0 \cdot 007$; Fig. $4(\mathrm{a})$ and (b)) and the macrophage marker CD68 ( $P=0 \cdot 001$; Fig. $4(\mathrm{c})$ and $(\mathrm{d}))$ in the SFA-HFD group

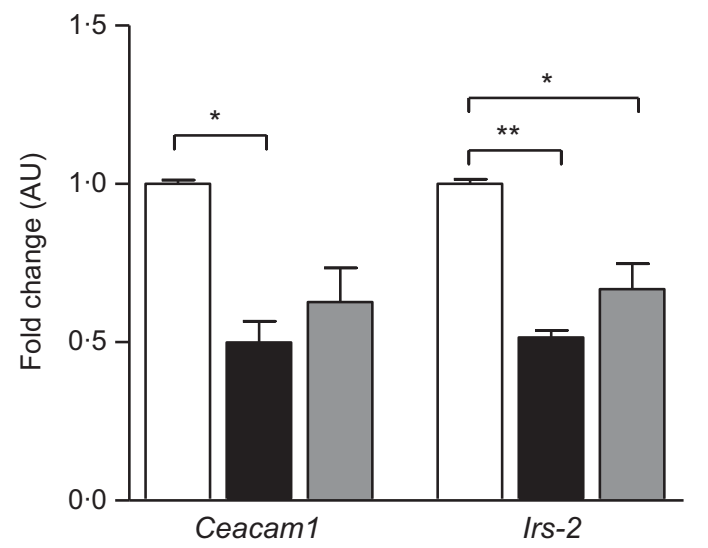

Fig. 3. Hepatic gene expression of Ceacam1 and Irs-2. ${ }^{*} P<0.05$, ${ }^{\star *} P<0.01$, ${ }_{* \star *} P<0.001$ between indicated comparisons by one-way ANOVA with Bonferroni post hoc comparisons. AU, arbitrary units; Ceacam1, carcinoembryonic antigen-related cell adhesion molecule 1 ; Irs-2, insulin receptor substrate 2. $\square$, Low-fat diet; $\square$, SFA-high-fat diet (HFD); $\square$, SFA-toMUFA-HFD. 
(a)

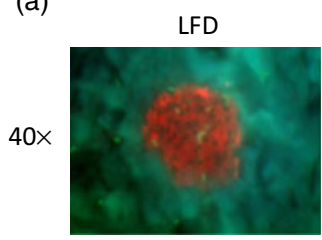

(c)

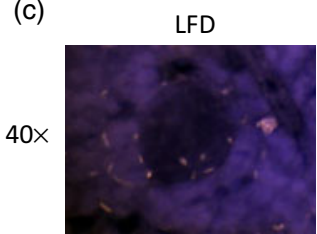

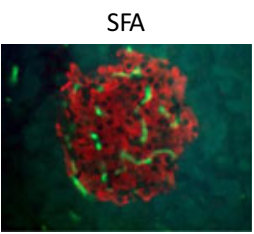

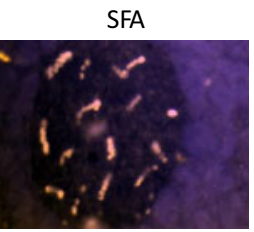

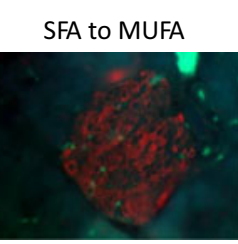

SFA to MUFA

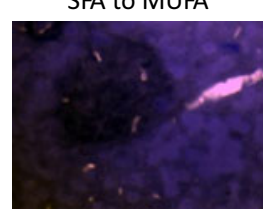

(b)

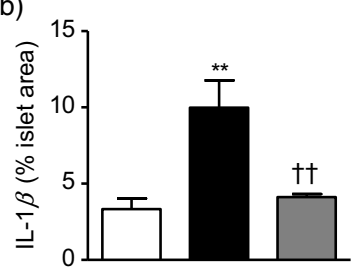

(d)

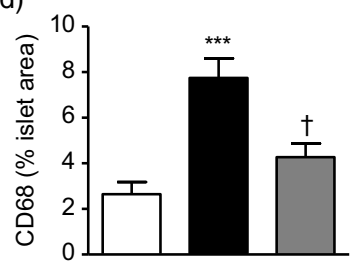

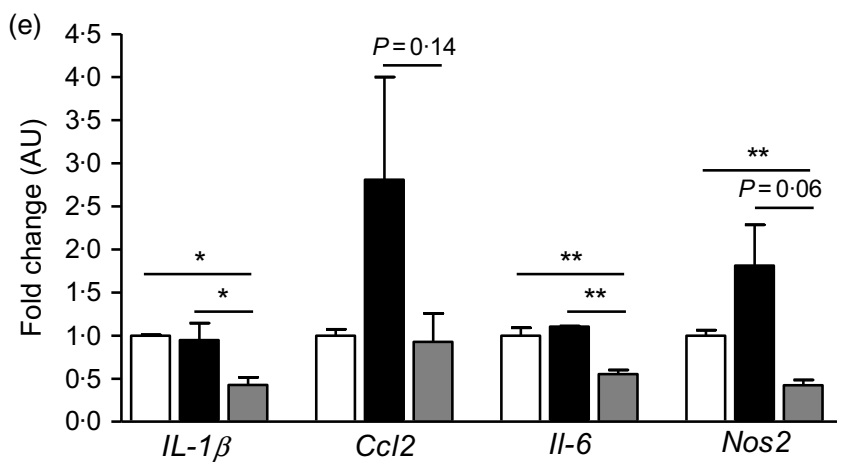

Fig. 4. Effects of a dietary switch from SFA-to-MUFA-high-fat diet (HFD) on pancreatic inflammatory markers. Immunostaining results for IL-1 $\beta$ (green) (a and b) and the macrophage marker CD68 (orange) (c and d). Expressions of inflammatory markers are shown in (e) ( $n$ 10). (b and d), ${ }^{* \star} P<0.01,{ }^{* \star *} P<0.001 v$. low-fat diet (LFD); $\dagger P<0.05, \dagger \dagger P<0.01$, SFA-HFD $v$. SFA-to-MUFA-HFD; (e), ${ }^{*} P<0.05$, ${ }^{\star \star} P<0.01$, between indicated comparisons by one-way ANOVA with Bonferroni post hoc comparisons. CD68, cluster of differentiation 68; AU, arbitrary units; Ccl2, monocyte chemoattractant protein 2; Nos2, nitric oxide synthase 2. (b, d, e) $\square$, LFD; HFD; $\square$, SFA-to-MUFA-HFD.

compared with the LFD group. Remarkably, elevated IL- $1 \beta$ and CD68 expression was attenuated when mice were switched from SFA-to-MUFA-HFD $(P=0.008$ and $0 \cdot 011$, respectively; Fig. 4(a)-(d)).

To validate the immunostaining results, we also determined islet gene expression of $I l-1 \beta, I l-6$ and Nos2. Islet gene expression of $I l-1 \beta, I l-6$ and Nos 2 was consistently lower in SFA-toMUFA-HFD mice compared with age-matched LFD and SFAHFD groups $(P=0.011 v$. LFD, $0.034 v$. SFA; $0.005 v$. LFD; and $P=0.001 v$. SFA; $0.003 v$. LFD, $0.057 v$. SFA, respectively; $n$ 10; Fig. 4(e)).

\section{Discussion}

Our study demonstrated that, in comparison with continuous consumption of SFA-HFD, switching to MUFA-HFD partially preserved the expression of markers of $\beta$-cell identity and differentiation, coincident with reduced pancreatic inflammation and attenuated impairments in islet function. We observed a very consistent pattern of changes in islet gene expression, wherein SFA-HFD significantly reduced markers of $\beta$-cell differentiation, proliferation and identity (e.g. Ins $2, N k x 6.1, N g n 3$ and $R f x 6$, and trends for $P d x 1$ and $P a x \sigma$ ), the down-regulation of which has been associated with impaired cell function ${ }^{(16,29)}$. Conversely, yet just as consistently, the same $\beta$-cell markers were not adversely affected after switching to MUFA-HFD and were similar to the LFD group. These findings extend previous in vitro work showing that MUFA exposure in human pancreatic $\beta$-cells prevented SFA-induced apoptosis and impairments in $\beta$-cell proliferation ${ }^{(15)}$. It appears that SFA-HFD weakens $\beta$-cell differentiation, whereas replacement of SFA with dietary MUFA prevents these detrimental effects and maintains differentiation potential at levels seen in healthy LFD-fed mice. Furthermore, the aforementioned effects likely contributed to the coinciding differences in islet insulin secretory capacity.

While our data suggest that fat quality may affect islet functionality, we need to acknowledge a potential impact of body weight. There was a small but significant difference in weight between SFA-HFD and SFA-to-MUFA-HFD groups at week 32. Nevertheless, when we weight-matched the insulin secretion response, there was a clear difference between groups based on fatty acid composition. In terms of potential differences between fatty acids, SFA, specifically palmitate, promotes inflammation in adipose tissue ${ }^{(5)}$ and pancreatic islets ${ }^{(15)}$. However, the impacts of different fatty acids are much less defined in the pancreas compared with adipose tissue ${ }^{(2)}$. We therefore assessed pancreatic inflammation with a view to understand the mechanisms driving SFA-HFD-induced impairments in islet gene expression. In this study, SFA-HFD significantly increased both IL- $1 \beta$ and the macrophage marker CD68 in islets, yet this effect was prevented in the SFA-to-MUFA-HFD group. 
Furthermore, changes in inflammatory gene expression mirrored immunostaining results, where MUFA-HFD reduced $I l-6, N o s 2$ and $I l-1 \beta$. Recent work by Nordmann et al. ${ }^{(28)}$ demonstrated in isolated islets that another common SFA, stearate, acted similarly to IL- $1 \beta$ and IL- 6 to significantly reduce markers of $\beta$-cell differentiation, including $P d x 1$ and $N k x 6.1$. Moreover, anti-inflammatory treatments, including anti-IL- $1 \beta$ antibody, anti-TNF $\alpha$ antibody and sodium salicylate, improved isolated islet insulin secretion ${ }^{(28)}$.

Our exvivo islet work extends and corroborates this concept of attenuating inflammation by dietary manipulation to protect islet biology. We demonstrated that a significant reduction in the insulin secretion stimulatory index from isolated islets of mice fed SFA-HFD was attenuated in mice switched from SFAto-MUFA-HFD. This concurs with the work of Maedler et al. ${ }^{(15)}$, which showed that ex vivo glucose-stimulated insulin secretion from human islets was completely abolished upon exposure to palmitate $(0.5 \mathrm{~mm}$ for $4 \mathrm{~d})$, whereas glucose-stimulated insulin secretion was completely restored by the addition of MUFA. Furthermore, Gerst et al. ${ }^{(30)}$ suggested that diabetogenic factors, including palmitate, target both pancreatic $\beta$-cells as well as pancreatic pre-adipocytes and adipocytes to promote inflammation, and this combination may accelerate $\beta$-cell failure. Taking these data together, we speculate that pancreatic inflammation observed in SFA-HFD-fed mice was attributable to the proinflammatory effects of pancreatic adipocytes and/or SFA-induced NLRP3-mediated IL-1 $\beta$ destruction of islet function ${ }^{(12,30)}$, which did not occur with the less inflammatory MUFA-enriched HFD.

Counterintuitively, we observed the presence of hyperinsulinaemia and enhanced in vivo insulin secretion response in SFA-HFD-fed mice despite $\beta$-cell dedifferentiation and reduced ex vivo islet insulin secretion; however, this may be explained by coincident inflammation. Indeed, genetic or diet-induced models of obesity contribute to both hyperinsulinaemia and pancreatic inflammation ${ }^{(31-36)}$. Through various mechanisms, including increased fibrosis ${ }^{(32)}$ or elevated islet blood perfusion ${ }^{(32,37)}$, inflammation in the pancreas can contribute to hyperinsulinaemia and downstream tissue dysfunction. Moreover, the elevated pancreatic IL- $1 \beta$ in SFA-HFD mice is fascinating in view of recent work suggesting that, although IL- $1 \beta$ is traditionally detrimental to islet function, it may also promote insulin secretion, highlighting the complexities of IL- $1 \beta$ functionality. Furthermore, IL-1 $\beta$ and systemic insulin appear to promote the secretion of one another ${ }^{(38)}$. Taken together, it's possible that despite dysfunctional insulin secretion in isolated islets, integrative in vivo biology maintains hyperinsulinaemia in SFA-HFD mice, emphasising the importance of using both ex vivo and in vivo models in animal interventions.

While the pancreas plays a pivotal role in systemic insulin homeostasis, the liver also regulates insulin clearance. The deletion of carcinoembryonic antigen-related cell adhesion molecule 1 (Ceacam1) $\left(\mathrm{CCl}^{-}\right)$causes hyperinsulinaemia due to impaired insulin clearance ${ }^{(26,27)}$. Typically, upon pancreatic insulin secretion, CEACAM1 associates with the insulin receptor and promotes hepatic insulin clearance and degradation ${ }^{(39)}$. Interestingly, SFA-HFD-fed mice had significantly reduced hepatic Ceacam 1 expression. Lester et al. ${ }^{(27)}$ demonstrated that feeding HFD ( $45 \%$ fat from lard) down-regulated hepatic Ceacam1 expression with hyperinsulinaemia.

Undoubtedly, this paper has not addressed all the possible mechanisms in relation to the potential protective effects of MUFA $v$. SFA-HFD on pancreatic function and insulin biology. The area of metabolic-inflammation is far more complex than previously anticipated ${ }^{(2)}$. It is not a simple paradigm, wherein proinflammatory cytokines impede metabolism, but that metabolic reconfiguration determines the nature of the cellular inflammatory profile ${ }^{(40)}$. For example, AMP-activated protein kinase $\alpha 1$ (AMPK) is a key regulator of NLRP3-mediated IL- $1 \beta$ activation $^{(41)}$. In this context, $A m p k$ attenuation in SFA-HFD islets concurrent with augmented pancreatic IL- $\beta$ and CD6 8 is noteworthy, since pancreatic AMPK may be necessary to maintain normal glucose-sensing and insulin secretion from $\beta$-cells ${ }^{(42)}$. Thus, elevated $A m p k$ in conjunction with lower pancreatic IL-1 $\beta$ in SFA-to-MUFA-HFD islets may partially protect against the overstimulation of $\beta$-cell insulin secretion and hyperinsulinaemia. Such bidirectional co-regulation of AMPK and IL- $1 \beta$ aligns with our previous work that focused on the adipose tissue, where MUFA-HFD preserved adipose AMPK and attenuated IL-1 $\beta$ activation compared with SFA-HFD ${ }^{(5)}$. A loss of $\beta$-cell AMPK can dysregulate differentiation and cause misexpression of key 'disallowed genes' (genes selectively repressed in $\beta$-cells), including $L d h a^{(25,42)}$. Therefore, lower islet $A m p k$ expression, concurrent with inflammation, may contribute to blunted $\beta$-cell differentiation in SFA-HFD-fed mice.

All studies have limitations; here a time course element would be insightful. Our islet experiments were only conducted at 32 weeks. Other studies following 8- or 14-week HFD feeding ${ }^{(32,33)}$ caused hyperinsulinaemia, islet inflammation and/or dysfunction, but islet insulin secretory function was still intact or elevated compared with controls. Contrary to this, a later time span reflects long-term dietary impact; 32 weeks was significantly longer to allow for the initial SFA-HFD insult before determining the impact of switching to MUFA-HFD. It is perhaps not surprising that islet secretory function became compromised in SFA-HFD islets after 32 weeks. Future work investigating islet function in response to prolonged diet intervention is warranted in a gender-dependent manner. It is also critical to acknowledge that we only investigated a palmitate-enriched SFA-HFD (16:0), whereas a previous work has shown that varying SFA chain lengths can have differential effects on obesogenic co-morbidities ${ }^{(43,44)}$. Moreover, switching from SFAto-MUFA-HFD did not resolve insulin resistance or the obese phenotype despite healthier islet function, further emphasising the complex effects of different fatty acid types on metabolic health. Further investigation into the effects of different SFA and MUFA types on pancreatic and whole-body health is warranted. Finally, further work is needed to support our mechanistic insights in order to unravel the precise molecular pathways underlying the differential effects of MUFA $v$. SFA. In particular, the exploration of pancreatic steatosis and/or inflammatory and apoptoticdependent pathways on $\beta$-cell mass and functionality, as well as the relative contribution of dietary fats on $\mathrm{CD} 68^{+}$immune cells $v$. $\beta$-cells, is warranted. 


\section{Conclusion}

In summary, this study highlights that switching to MUFA-HFD prevented further progression of SFA-HFD-induced inflammation in pancreatic islets. MUFA-HFD partly attenuated hyperinsulinaemia compared with SFA-HFD, an important consideration since HFD-induced hyperinsulinaemia may further drive obesityrelated complications. Collectively, this work highlights that changing the type of dietary fat may have significant implications on pancreatic function and health. While the translational potential needs to be verified in human populations, recent retrospective analyses ${ }^{(4,45)}$ suggest that dietary fat reconfiguration may have a potential to differentially modulate the progression of insulin resistance and diabetes in man.

\section{Acknowledgements}

The authors thank Catherine Moss of the Conway Institute of Biomolecular and Biomedical Research, as well as the biomedical facility staff at the University College Dublin for their technical support.

This work was supported by Science Foundation Ireland $(\mathrm{H}$. M. R., grant no. SFI PI 11/PI/1119), Enterprise Ireland (H. M. R., grant no. TC2013-0001), the Irish Department of Agriculture, Food and the Marine (H. M. R., grant no. 14/F/828 'ImmunoMet') and the Joint Programming Initiative Healthy Diet for a Healthy Life FOODBALL (The Food Biomarkers Alliance) Programme (H. M. R., grant no. 14/JPI-HDHL/B3076). G. A. R. is supported by MRC Programme (MR/J0003042/1, MR/ N00275X/1, MR/L020149/1 (DIVA)), Wellcome Trust Senior Investigator (WT098424AIA) and Diabetes UK Project (BDA11/ 0004210, BDA/15/0005275) grants. The funding bodies had no role in the design, analysis or writing of this article.

J. C. R. conducted research and co-wrote the manuscript. M.-S. N.-T. conducted research and edited the manuscript. C. L. L., A. A. C., A. M. M., A. F. and O. M. F. carried out research during the study. F. C. M. reviewed the data and edited the manuscript. G. A. R. co-designed the study, reviewed the data and edited the manuscript. H. M. R. designed the study, reviewed the data and co-wrote the manuscript.

G. A. R. has received grant funding from Les Laboratoires Serviers.

\section{Supplementary material}

For supplementary materials referred to in this article, please visit https://doi.org/10.1017/S0007114520000859

\section{References}

1. Mehran AE, Templeman NM, Brigidi GS, et al. (2012) Hyperinsulinemia drives diet-induced obesity independently of brain insulin production. Cell Metab 16, 723-737.

2. Ralston JC, Lyons CL, Kennedy EB, et al. (2017) Fatty acids and NLRP3 inflammasome - mediated inflammation in metabolic tissues. Annu Rev Nutr 37, 77-102.

3. Murphy AM, Lyons CL, Finucane OM, et al. (2015) Interactions between differential fatty acids and inflammatory stressorsimpact on metabolic health. Prostaglandins Leukot Essent Fatty Acids 92, 49-55.
4. Gulseth HL, Gjelstad IMF, Tiereny AC, et al. (2019) Effects of dietary fat on insulin secretion in subjects with the metabolic syndrome. Eur I Endocrinol 180, 321-328.

5. Finucane OM, Lyons CL, Murphy AM, et al. (2015) Monounsaturated fatty acid - enriched high-fat diets impede adipose NLRP3 inflammasome - mediated IL-1 $\beta$ secretion and insulin resistance despite obesity. Diabetes 64, 2116-2128.

6. Kien CL, Bunn JY, Fukagawa NK, et al. (2015) Lipidomic evidence that lowering the typical dietary palmitate to oleate ratio in humans decreases the leukocyte production of proinflammatory cytokines and muscle expression of redoxsensitive genes. J Nutr Biochem 26, 1599-1606.

7. Kien CL, Bunn JY, Poynter ME, et al. (2013) A lipidomics analysis of the relationship between dietary fatty acid composition and insulin sensitivity in young adults. Diabetes 62, 1054-1063.

8. Kraakman MJ, Kammoun HL, Allen TL, et al. (2015) Blocking IL-6 trans-signaling prevents high-fat diet-induced adipose tissue macrophage recruitment but does not improve insulin resistance. Cell Metab 21, 403-416.

9. Maedler K, Sergeev P, Ris F, et al. (2002) Glucose-induced $\beta$ cell production of IL- $1 \beta$ contributes to glucotoxicity in human pancreatic islets. J Clin Invest 110, 851-860.

10. Donath MY, Böni-Schnetzler M, Ellingsgaard H, et al. (2010) Cytokine production by islets in health and diabetes: cellular origin, regulation and function. Trends Endocrinol Metab 21, 261-267.

11. Spinas GA, Palmer JP, Mandrup-Poulsen T, et al. (1988) The bimodal effect of interleukin 1 on rat pancreatic beta-cells stimulation followed by inhibition - depends upon dose, duration of exposure, and ambient glucose concentration. Acta Endocrinol 119, 307-311.

12. Masters SL, Dunne A, Subramanian SL, et al. (2010) Activation of the Nlrp3 inflammasome by islet amyloid polypeptide provides a mechanism for enhanced IL- $1 \beta$ in type 2 diabetes. Nat Immunol 11, 897-904.

13. Reynolds CM, McGillicuddy FC, Harford KA, et al. (2012) Dietary saturated fatty acids prime the NLRP3 inflammasome via TLR4 in dendritic cells - implications for diet-induced insulin resistance. Mol Nutr Food Res 56, 1212-1222.

14. Wen H, Gris D, Lei Y, et al. (2011) Fatty acid-induced NLRP3PYCARD inflammasome activation interferes with insulin signaling. Nat Immunol 12, 408-415.

15. Maedler K, Oberholzer J, Bucher P, et al. (2003) Monounsaturated fatty acids prevent the deleterious effects of palmitate and high glucose on human pancreatic $\beta$-cell turnover and function. Diabetes 52, 726-733.

16. Rutter GA, Pullen TJ, Hodson DJ, et al. (2015) Pancreatic $\beta$-cell identity, glucose sensing and the control of insulin secretion. Biochem J 466, 203-218.

17. Ravier MA \& Rutter GA (2010) Isolation and culture of mouse pancreatic islets for ex vivo imaging studies with trappable or recombinant fluorescent probes. In Mouse Cell Culture: Methods and Protocols, pp. 171-184 [A Ward and D Tosh, editors]. Totowa, NJ: Humana Press.

18. Leclerc I, Woltersdorf WW, da Silva Xavier G, et al. (2004) Metformin, but not leptin, regulates AMP-activated protein kinase in pancreatic islets: impact on glucose-stimulated insulin secretion. Am J Physiol Endocrinol Metab 286, E1023-E1031.

19. Mitchell RK, Nguyen-Tu M-S, Chabosseau P, et al. (2017) The transcription factor Pax6 is required for pancreatic $\beta$ cell identity, glucose-regulated ATP synthesis, and $\mathrm{Ca}(2+)$ dynamics in adult mice. I Biol Chem 292, 8892-8906.

20. Carrat GR, Hu M, Nguyen-Tu M-S, et al. (2017) Decreased STARD10 expression is associated with defective insulin secretion in humans and mice. Am J Hum Genet 100, 238-256. 
21. Ralston JC \& Mutch DM (2015) SCD1 inhibition during 3T3-L1 adipocyte differentiation remodels triacylglycerol, diacylglycerol and phospholipid fatty acid composition. Prostaglandins Leukot Essent Fatty Acids 98, 29-37.

22. The Oxford Centre for Diabetes, Endocrinology and Metabolism (2017) Diabetes trial unit HOMA2 calculator. http://www.dtu.ox.ac.uk/homacalculator/index.php

23. Sekine N, Cirulli V, Regazzi R, et al. (1994) Low lactate dehydrogenase and high mitochondrial glycerol phosphate dehydrogenase in pancreatic beta-cells. Potential role in nutrient sensing. J Biol Chem 269, 4895-4902.

24. Pullen TJ, Huising MO \& Rutter GA (2017) Analysis of purified pancreatic islet beta and alpha cell transcriptomes reveals $11 \beta$-hydroxysteroid dehydrogenase (Hsd11b1) as a novel disallowed gene. Front Genet 8, 41.

25. Kone M, Pullen TJ, Sun G, et al. (2014) LKB1 and AMPK differentially regulate pancreatic $\beta$-cell identity. FASEB J $\mathbf{2 8}$ 4972-4985.

26. Huang J, Ledford KJ, Pitkin WB, et al. (2013) Targeted deletion of murine CEACAM 1 activates PI3K-Akt signaling and contributes to the expression of (Pro)renin receptor via CREB family and NF- $\mathrm{\kappa B}$ transcription factors. Hypertension $\mathbf{6 2}, 317-323$.

27. Lester SG, Russo L, Ghanem SS, et al. (2015) Hepatic CEACAM1 over-expression protects against diet-induced fibrosis and inflammation in white adipose tissue. Front Endocrinol 6, 116

28. Nordmann TM, Dror E, Schulze F, et al. (2017) The role of inflammation in $\beta$-cell dedifferentiation. Sci Rep 7, 6285.

29. Swisa A, Glaser B \& Dor Y (2017) Metabolic stress and compromised identity of pancreatic beta cells. Front Genet $\mathbf{8}, 21$.

30. Gerst F, Wagner R, Kaiser G, et al. (2017) Metabolic crosstalk between fatty pancreas and fatty liver: effects on local inflammation and insulin secretion. Diabetologia 60, 2240-2251.

31. Dawson DW, Hertzer K, Moro A, et al. (2013) High fat, high calorie diet promotes early pancreatic neoplasia in the conditional kras(G12D) mouse model. Cancer Prev Res 6, 1064-1073.

32. Pettersson US, Waldén TB, Carlsson P-O, et al. (2012) Female mice are protected against high-fat diet induced metabolic syndrome and increase the regulatory $\mathrm{T}$ cell population in adipose tissue. PLOS ONE 7, e46057.

33. Reimer MK \& Ahrén B (2002) Altered $\beta$-cell distribution of pdx-1 and GLUT-2 after a short-term challenge with a highfat diet in C57BL/6J mice. Diabetes 51, S138-S143.
34. Delghingaro-Augusto V, Madad L, Chandra A, et al. (2014) Islet inflammation, hemosiderosis, and fibrosis in intrauterine growth-restricted and high fat-fed Sprague-Dawley rats. Am J Pathol 184, 1446-1457.

35. Venkatesan V, Madhira SL, Malakapalli VM, et al. (2013) Obesity, insulin resistance, and metabolic syndrome: a study in WNIN/Ob rats from a pancreatic perspective. BioMed Res Int 2013, 617569.

36. Singh H, Ganneru S, Malakapalli V, et al. (2014) Islet adaptation to obesity and insulin resistance in WNIN/GR-Ob rats. Islets $\mathbf{6}$, e998099.

37. Svensson AM, Hellerström C \& Jansson L (1996) Diet-induced obesity and pancreatic islet blood flow in the rat: a preferential increase in islet blood perfusion persists after withdrawal of the diet and normalization of body weight. J Endocrinol 151, 507-511.

38. Dror E, Dalmas E, Meier DT, et al. (2017) Postprandial macrophage-derived IL- $1 \beta$ stimulates insulin, and both synergistically promote glucose disposal and inflammation. Nat Immunol 18, 283.

39. Poy MN, Yang Y, Rezaei K, et al. (2002) CEACAM1 regulates insulin clearance in liver. Nat Genet 30, 270-276.

40. Jha AK, Huang SC-C, Ergushichev A, et al. (2015) Network integration of parallel metabolic and transcriptional data reveals metabolic modules that regulate macrophage polarization. Immunity 42, 419-430.

41. O'Neill LAJ \& Hardie DG (2013) Metabolism of inflammation limited by AMPK and pseudo-starvation. Nature $\mathbf{4 9 3}$, 346-355.

42. Beall C, Piipari K, Al-Qassab H, et al. (2010) Loss of AMPactivated protein kinase $\alpha 2$ subunit in mouse $\beta$-cells impairs glucose-stimulated insulin secretion and inhibits their sensitivity to hypoglycaemia. Biochem J 429, 323-333.

43. Žáček P, Bukowski M, Mehus A, et al. (2019) Dietary saturated fatty acid type impacts obesity-induced metabolic dysfunction and plasma lipidomic signatures in mice. J Nutr Biochem $\mathbf{6 4}$, 32-44.

44. St-Onge M-P \& Jones PJH (2002) Physiological effects of medium-chain triglycerides: potential agents in the prevention of obesity. J Nutr 132, 329-332.

45. Yubero-Serrano EM, Delgado-Lista J, Tierney AC, et al. (2015) Insulin resistance determines a differential response to changes in dietary fat modification on metabolic syndrome risk factors: the LIPGENE study. Am J Clin Nutr 102, 1509-1517. 